\title{
ОПЫТ ПРИМЕНЕНИЯ ПОЛНОЭКЗОМНОГО СЕКВЕНИРОВАНИЯ В ДИАГНОСТИКЕ НАСЛЕДСТВЕННЫХ СИНДРОМОВ С ЭНДОКРИННЫМИ НАРУШЕНИЯМИ
}

'Гусева М.В., ${ }^{1}$ Мусатова Е.В., 'Хмелькова Д.Н., ${ }^{1}$ Котов И.Н., ${ }^{2}$ Толмачева Е.Р., ${ }^{2}$ Коновалов Ф.А., ${ }^{1,3}$ Поволоцкая И.С., 'Миронова И.В., 'Каймонов В.С., 'Померанцева Е.А.

\author{
'ООО «Центр генетики и репродуктивной медицины «ГЕНЕТИКО», Москва \\ ${ }^{2}$ «Лаборатория клинической биоинформатики», Москва

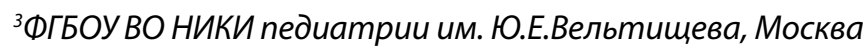

Группа наследственных заболеваний с эндокринными проявлениями представлена широким спектром гетерогенной патологии. При этом клинически некоторые нозологические формы могут быть трудноотличимы друг от друга. В последнее время достижения в области медицинских знаний и молекулярно-генетических технологий позволили проводить эффективное генетическое тестирование с целью уточнения диагноза. Полноэкзомное секвенирование является современным высокопроизводительным методом генетического исследования, позволяющим выявлять генетические нарушения, встречающиеся В том числе при эндокринной патологии. В настоящем исследовании представлено описание использования полноэкзомного секвенирования с целью поиска каузативного генетического варианта у пациентов С эндокринными нарушениями.

МАТЕРИАЛЫ И МЕТОДЫ: представлено 4 клинических случая. Все обследуемые имели явные или скрытые нарушения в эндокринном статусе. Возраст пациентов составил от 2 до 12 лет. Пациенты №1-3 - лица мужского пола, №4 - женского. Полноэкзомное секвенирование проводилось на платформе NovaSeq 6000 (Illumina) с длиной прочтения 2*100 п.о. ДНК-библиотеки были получены с помощью набора SureSelectXTHumanAllExonV7 (Agilent).

PЕЗУЛЬТАТЫ: в результате проведения полноэкзомного секвенирования были выявлены варианты, объясняющие клинические проявления у пациентов. Пациент 1 не предъявлял активных жалоб на нарушение эндокринного статуса, но у него был выявлен вариант в гене андрогенного рецептора. У пациента 2 направительный диагноз (нейрофиброматоз) частично объяснял наблюдаемую клиническую картину. При этом в результате полноэкзомного секвенирования был выявлен вариант в гене, ассоциированном с гипопаратиреозом. Данный синдром иногда ошибочно может быть принят за факоматоз. У пациента 3 был выявлен вариант в гене, который ассоциирован с развитием заболевания с отсроченными проявлениями: для аутоиммунного полиэндокринного синдрома, тип I характерно появление новых симптомов до 5-го десятилетия жизни. У пациента 4 был заподозрен синдром Барде-Бидля, что было подтверждено данными полноэкзомного секвенирования.

ВЫВоды: применение полноэкзомного секвенирования помогает поставить точный диагноз в сложных клинических ситуациях, например, у пациентов, имеющих эндокринные нарушения при стертой клинической картине и множественных проявлениях со стороны других органов и систем. Но в ряде случаев определение каузативности найденных вариантов требует проведения дополнительного обследования с помощью применения других методов диагностики.

КЛЮЧЕВЫЕ СЛОВА: эндокринные нарушения, полноэкзомное секвенирование, NGS, гипопаратиреоз, синдром БардеБидля. 